\title{
MiR-21-5p enhances the progression and paclitaxel resistance in drug-resistant breast cancer cell lines by targeting PDCD4
}

\author{
L. TAO ${ }^{1}$, Y. Q. WU' 1 , S. P. ZHANG ${ }^{2, *}$ \\ ${ }^{1}$ Department of Breast Surgery, Chifeng Municipal Hospital, Chifeng, Inner Mongolia, China; ${ }^{2}$ Department of Breast and Thyroid Surgery, \\ Union Hospital, Tongji Medical College, Huazhong University of Science and Technology, Wuhan, Hubei, China
}

*Correspondence: majdoctorhn@sina.com

Received December 7, 2018 / Accepted March 20, 2019

\begin{abstract}
MiR-21-5p has been identified as an oncogene to enhance human tumor progression. Here, we explored the mechanism by which miR-21-5p regulated progression and paclitaxel (PTX) resistance in drug-resistant breast cancer (BC) cell lines. qRT-PCR assays were used to assess the expression levels of miR-21-5p and PDCD4 mRNA, and western blotting was used to detect PDCD4 protein level in PTX-resistant BC cell lines. Dual-luciferase reporter assay was used to observe the

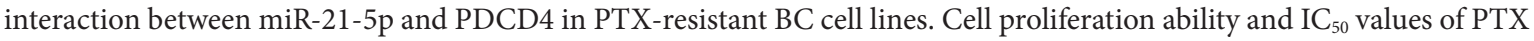
were measured by CCK- 8 assay, cell cycle progression and apoptosis were determined with flow cytometry analysis, and cell migration and invasion capacities were analyzed using Transwell assay. Xenograft mice assay was used to validate the important role of miR-21-5p as a regulator on PTX-resistance BC cells growth in vivo. Then, we found that miR-21-5p was upregulated and PDCD4 was downregulated in BC tissues and PTX-resistant BC cell lines. MiR-21-5p silencing or PDCD4 overexpression ameliorated PTX resistance and inhibited the progression in PTX-resistant BC cell lines. Moreover, PDCD4 was demonstrated to be a direct target of miR-21-5p. MiR-21-5p exerted its regulatory effect by PDCD4 in PTX-resistant $\mathrm{BC}$ cell lines. Additionally, miR-21-5p silencing inhibited tumor growth in vivo. Therefore, our study demonstrated that miR-21-5p silencing ameliorated PTX resistance and inhibited the progression in PTX-resistant BC cell lines at least partly by targeting PDCD4, providing miR-21-5p as an effective therapeutic target for PTX-resistant BC treatment.
\end{abstract}

Key words: breast cancer (BC), miR-21-5p, programmed cell death 4 (PDCD4), paclitaxel-resistance

Breast cancer (BC), the most prevalent malignancy in women, is the second leading cause of cancer-death among women after lung cancer worldwide [1]. Due to the improvement of systemic therapeutic methods, such as surgical resection, chemotherapy and radiotherapy, the mortality of $\mathrm{BC}$ has substantially decreased in recent years [2]. However, distal metastasis and chemoresistance are still the main obstacles in BC treatment [3]. Therefore, it is of importance to identify more effective therapeutic targets for BC treatment.

MicroRNAs (miRNAs), a type of small non-coding, single-stranded RNAs of 18-23 nucleotides, play important roles in multiple physiopathology pathways [4]. MiRNAs function as negative regulators of gene expression by binding to 3'-untranslated region (UTR) of target-mRNA, resulting in translational repression and target mRNA degradation [4]. Growing amount of evidence has suggested that miRNA dysregulation is tightly involved in tumorigenesis, cancer progression and chemoresistance [5]. MiR-21-5p has been identified as an oncogene given its abilities to repress the actions of several tumor suppressor genes and to enhance tumor progression in esophageal cancer [6], Hodgkin lymphoma [7], lung cancer [8] and colon adenocarcinoma [9]. Moreover, miR-21 expression was elevated in BC tissues and miR-21 upregulation promoted the tumor progression and associated with poor disease-free survival [10]. Additionally, miR-21 depletion led to a reduction of chemoresistance against gemcitabine in pancreatic cancer and cholangiocarcinoma cell lines $[11,12]$.

The tumor suppressor programmed cell death 4 (PDCD4) is widely acknowledged to suppress tumorigenesis and progression in human cancers [13]. High PDCD4 expression was demonstrated to be sufficient to promote paclitaxel (PTX) sensitivity in multiple human cancer cell lines [14]. Moreover, PDCD4 was reported as a functional target of miR-21 in BC and colorectal cancer cells $[15,16]$. Additionally, a previous paper illuminated that miR-21 enhanced 5-fluorouracil resistance in pancreatic cancer cells through targeting PDCD4 [17]. However, the effect of inter- 
play between miR-21-5p and PDCD4 in PTX-resistant BC cell lines remains undefined. In this study, we found that miR-21-5p was upregulated, and PDCD4 was downregulated in BC tissues and PTX-resistant BC cell lines. Furthermore, miR-21-5p silencing ameliorated PTX resistance and inhibited the progression in PTX-resistant BC cell lines at least partly through targeting PDCD4.

\section{Materials and methods}

Tissues collection. Twenty pairs of BC tissues and adjacent nontumor tissues were obtained from Chifeng Municipal Hospital (Chifeng, Inner Mongolia, China). All patients did not receive any therapy before surgery. All tissues were stored at $-80^{\circ} \mathrm{C}$. Informed consent was obtained from all patients, and the study was approved by the Ethics Committee of Chifeng Municipal Hospital.

Cell culture and treatment. Human mammary epithelial cell line (MCF-10A) and BC cell lines (MCF-7 and MDA-MB-231) were purchased from ATCC (Manassas, VA, USA), and cultured in RPMI-1640 medium (Thermo Fisher Scientific, Waltham, MA, USA) containing L-glutamine, supplemented with $10 \%$ FBS (Thermo Fisher Scientific) and $1 \%$ penicillin/streptomycin (Thermo Fisher Scientific) in a humidifier chamber at $37^{\circ} \mathrm{C}$ with $5 \% \mathrm{CO}_{2}$.

PTX-resistant BC cell lines (MCF-7/PTX and MDA-MB231/PTX) were constructed by sequential exposure to PTX (Sigma-Aldrich, St. Louis, MO, USA) at stepwise increasing concentrations over a period of 6 months, as described previously [18]. Additional $1 \mu \mathrm{M}$ PTX was added into the culture medium to maintain PTX-resistant cell line drug-resistant phenotype.

Cell transfection. PDCD4 overexpression plasmid (pcDNA-PDCD4) was constructed by GenePhama (Shanghai, China), and pcDNA-NC was used as negative control. The modified miR-21-5p mimics, miR-21-5p inhibitors (anti-miR-21-5p), siRNA targeting PDCD4 (si-PDCD4) or corresponding controls (miR-NC mimics, anti-miR-NC or si-NC) were designed and synthetized by GenePhama. Cells were transfected with $100 \mathrm{nM}$ of the indicated oligonucleotide or $10 \mathrm{ng}$ of plasmid using Lipofectamine 3000 Reagent (Invitrogen, Carlsbad, CA, USA) according to the manufacturer's protocol.

RNA isolation and quantitative real-time PCR (qRT-PCR). qRT-PCR assays were used to assess the expression levels of miR-21-5p and PDCD4 mRNA in BC tissues and cells. For PDCD4 mRNA level detection, total RNA was extracted from tissues and cells with $\mathrm{TRIzol}^{\mathrm{TM}}$ Reagent (Invitrogen) and reversely transcribed into cDNA with M-MLV reverse transcriptase (Promega, Madison, WI, USA). Then, qRT-PCR assay was performed using SYBR green master mix (Roche Diagnostics, Mannheim, Germany) on an ABI 7900 system (Applied Biosystems, Foster City, CA, USA). The relative expression of PDCD $4 \mathrm{mRNA}$ was calculated by $2^{-\Delta \Delta C t}$ method and normalized to that of GAPDH.
For miR-21-5p expression measurement, total RNA was isolated with TRIzol $^{\mathrm{m}}$ Reagent, reverse-transcribed using a TaqMan Reverse Transcription Kit (Applied Biosystems), and subjected to qRT-PCR using a TaqMan MicroRNA Assay Kit (Life Technology, Grand Island, NY). $2^{-\Delta \Delta \mathrm{Ct}}$ method was used to calculate miR-21-5p expression and U6 was used as endogenous control.

CCK-8 assay of cell proliferation and $\mathrm{IC}_{50}$ values. Cell proliferation ability and $\mathrm{IC}_{50}$ values of cell to PTX were detected using Cell Count-8 Kit (CCK-8, Dojindo Laboratories, Kumamoto, Japan) according to the manual of manufacturer. For the measurement of cell proliferation, at $0,24,48$, 72 and $96 \mathrm{~h}$ post-transfection, $10 \mu \mathrm{l}$ of CCK- 8 solution was added into each well of 96 -well plates at $4^{\circ} \mathrm{C}$ for $2 \mathrm{~h}$, followed by the detection of the absorbance at the wavelength of $450 \mathrm{~nm}$. For the determination of $\mathrm{IC}_{50}$ values, transfected cells were exposed to different concentration $(0,2,5,10$, $20 \mu \mathrm{M}$ ) of PTX for $48 \mathrm{~h}$, followed by the measurement of $\mathrm{IC}_{50}$ values of PTX by CCK-8 assay.

Flow cytometry analysis of cell cycle progression and apoptosis. For cell cycle analysis, flow cytometry was performed using EZCell ${ }^{\mathrm{Tm}}$ Cell Cycle Analysis Kit (BioVision, San Francisco, CA, USA). Briefly, $48 \mathrm{~h}$ after transfection, cells were stained with $50 \mu \mathrm{g} / \mathrm{ml}$ propidium iodide (PI), followed by determination of cell cycle distribution with a flow cytometer (Epics XLM.CL, Beckman Coulter, Fullerton, CA, USA).

For cell apoptosis analysis, flow cytometry analysis was performed with Annexin V-FITC/PI Apoptosis Detection Kit (BD Bioscience, Franklin Lakes, NJ, USA). To be brief, at $48 \mathrm{~h}$ post-transfection, cells were stained with indicated Annexin V-FITC and PI, followed by the measurement of cell apoptosis using a flow cytometer.

Transwell assay of cell migration and invasion. Cell migration and invasion capacities were detected by Transwell assays. For migration assay, $200 \mu \mathrm{l}$ of serum-free medium containing $2.0 \times 10^{4}$ cells were seeded into the upper chamber with the non-coated membrane in 24-Transwell plate $(8 \mu \mathrm{m}$ pore size, Corning, Toledo, NY, USA). For invasion analysis, $2.0 \times 10^{4}$ cells maintained in serum-free medium were seeded into the upper chamber with the Matrigel-coated membrane (Corning). In both assays, $500 \mu \mathrm{l}$ of growth medium supplemented with $10 \%$ FBS were added into the lower chamber. After incubation for $48 \mathrm{~h}$, migrated or invaded cells to the lower surface of membrane were fixed with $4 \%$ paraformaldehyde (Sigma-Aldrich) and stained with $0.1 \%$ crystal violet (Sigma-Aldrich). Images were taken with ImageJ software (National Institutes of Health, Bethesda, Maryland, USA) and cell number was counted under a Nikon microscope (Shinagawa, Tokyo, Japan).

Western blotting. For total protein extraction, cells were lysed with the lysis buffer $(50 \mathrm{mM}$ Tris- $\mathrm{HCl}, \mathrm{pH}=7.4$, $150 \mathrm{mM} \mathrm{NaCl}, 1 \%$ Triton X-100, 1 mM EDTA, 1\% sodium deoxycholate, $0.1 \%$ SDS) supplemented with protease and phosphatase inhibitors (Roche Diagnostics). Protein concentration was measured using BCA Protein Assay Kit (Thermo 
Fisher Scientific). $50 \mu \mathrm{g}$ of protein lysates were separated by $10 \%$ SDS-PAGE and then transferred to PVDF membranes (Bio-Rad, Hertfordshire, UK). After blocking with 5\% non-fat milk, the membranes were incubated with antiPDCD4 (1:1000, Cell Signaling Technology, Danvers, MA, USA), anti-E-cadherin (1:1000, Cell Signaling Technology), anti-Vimentin (1:1000, Cell Signaling Technology) and anti- $\beta$-actin (1:1000, Cell Signaling Technology) primary antibody, followed by an incubation with a horseradish peroxidase-conjugated secondary antibody (1:1000, Cell Signaling Technology). Protein bands were visualized using a ECL plus Western Blotting Detection Kit (GE Healthcare, Chalfont St Giles, UK) with Biorad Flour S Imager (Bio-Rad).
Dual-luciferase reporter assay. Dual-luciferase reporter assay was performed to confirm whether PDCD4 was a direct target of miR-21-5p in PTX-resistant BC cells. $100 \mathrm{ng}$ of PDCD4 wild-type or mutant-type reporter plasmid (PDCD4 3'-UTR-WT or PDCD4 3'-UTR-MUT) constructed by GenePhama were transfected into cells together with $100 \mathrm{nM}$ of miR-NC mimics, miR-21-5p mimics, anti-miR-NC or anti-miR-21-5p. $48 \mathrm{~h}$ after transfection, the luciferase activity was measured using the Dual-Luciferase Reporter Assay System (Promega).

Xenograft mice assay in vivo. Male $\mathrm{BALB} / \mathrm{c}$ nude mice (6-8 weeks old) were purchased from Henan Research Center of Laboratory Animal (Zhengzhou, Henan, China) and bred
A

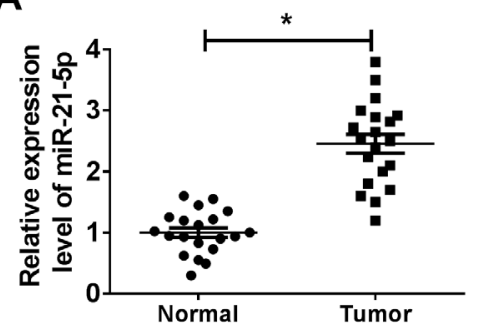

C

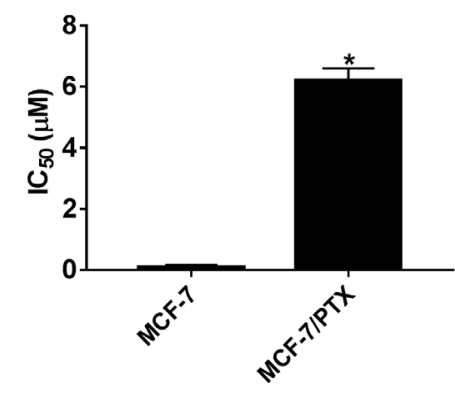

E

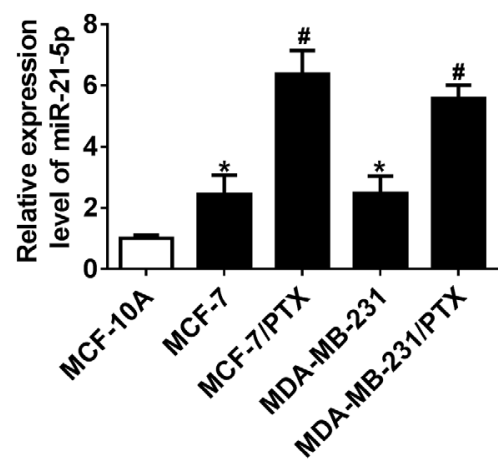

B

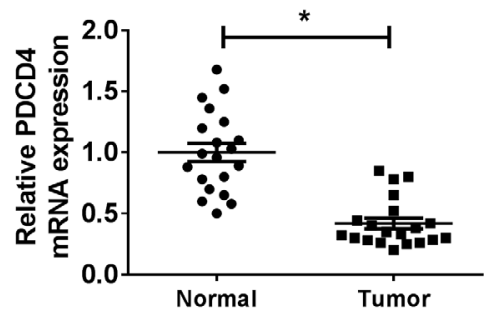

D

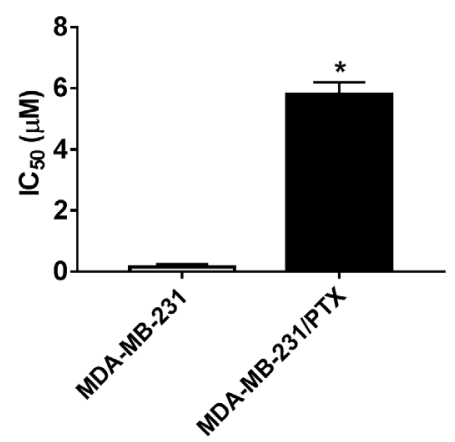

$\mathbf{F}$

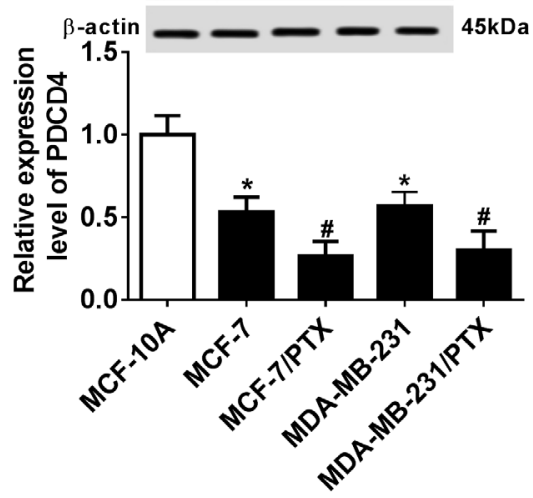

Figure 1. MiR-21-5p was upregulated and PDCD4 was downregulated in BC tissues and PTX-resistant BC cell lines. The expression levels of miR-21-5p (A) and PDCD4 mRNA (B) were detected by QRT-PCR assay in 20 pairs of BC tissues and adjacent non-tumor tissues. MCF-7 (C) and MDA-MB-231 (D) cells were induced by PTX with stepwise increased concentration over a period of 6 months to establish stable PTX-resistant cells, and then PTX-

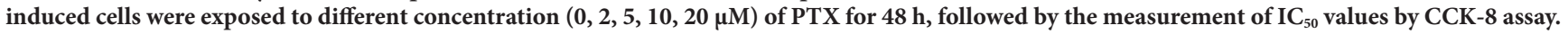
qRT-PCR assay of miR-21-5p expression (E) and western blotting analysis of PDCD4 expression (F) in MCF-7/PTX, MDA-MB-231/PTX cells and their parental cells. ${ }^{*} \mathrm{p}<0.05$ or ${ }^{*} \mathrm{p}<0.05$ vs. MCF-10A, MCF-7 or MDA-MB-231. 
in special-pathogen-free condition. About $5.0 \times 10^{6} \mathrm{MCF}-7 /$ PTX cells were subcutaneously injected into nude mice. 2 days after injection, intratumoral injection of anti-miR-NC or anti-miR-21-5p was performed every 4 days $(n=10)$. Also, 5 days after injection, an intravenous administration of PTX solution $(1 \mathrm{mg} / \mathrm{kg})$ in each mouse was performed every 5 days. 35 days after injection, all mice were euthanized and tumor tissues were excised for qRT-PCR and western blotting assays. All animal experiments were carried out following the national standard of the Care and Use of Laboratory Animals and our study was approved by the Institutional Committee of Chifeng Municipal Hospital.

Statistical analysis. All data were analyzed using SPSS 17.0 (SPSS Inc., Chicago, IL, USA) and were presented as mean \pm standard error of mean (SEM). Student's t-text was performed to compare the difference of two groups. A p-value of less than 0.05 was considered statistically significant.
Results

MiR-21-5p was upregulated and PDCD4 was downregulated in BC tissues and PTX-resistant BC cell lines. Firstly, we detected the expression of miR-21-5p and PDCD4 in BC tissues and adjacent non-tumor tissues. qRT-PCR results showed that compared with control tissues, miR-21-5p expression was significantly increased in BC tissues (Figure 1A). On the contrary, PDCD4 mRNA expression was markedly decreased in BC tissues (Figure 1B). To establish stable PTX-resistant cell lines, MCF-7 and MDA-MB-231 cells were induced by PTX with stepwise increased concentration over a period of 6 months. As shown in Figures $1 \mathrm{C}$ and $1 \mathrm{D}$, the $\mathrm{IC}_{50}$ values of MCF-7/PTX and MDA-MB-231/PTX cells were markedly elevated by PTX induction compared with their parental cells, indicating the successful establishment of PTX-resistant cell lines. Subsequently, we assessed
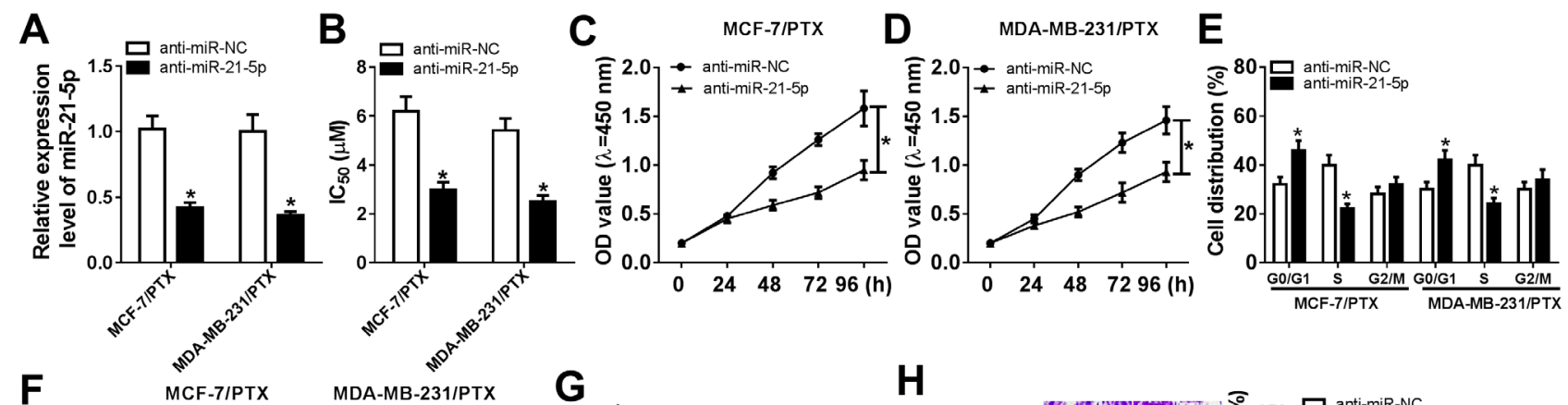

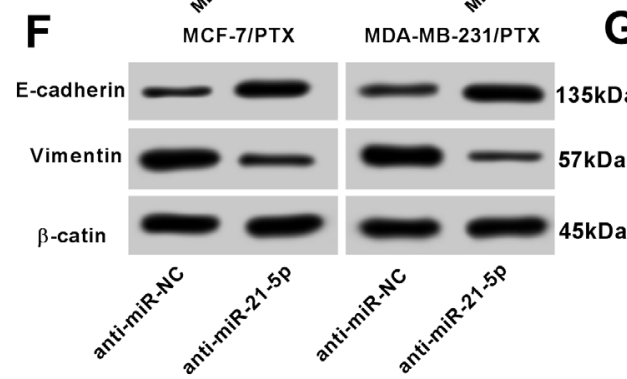

I

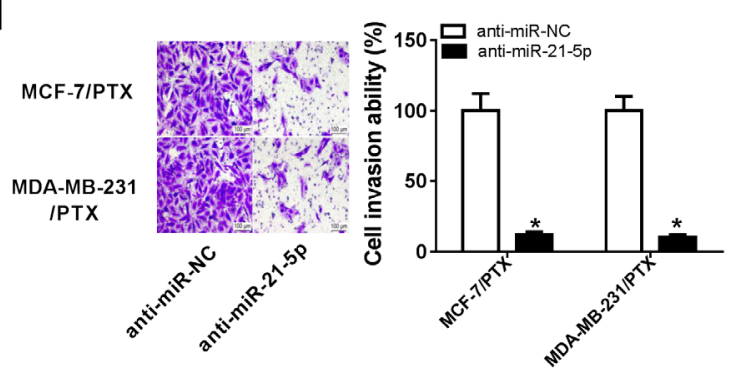

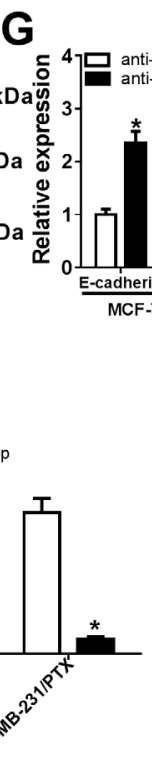

H

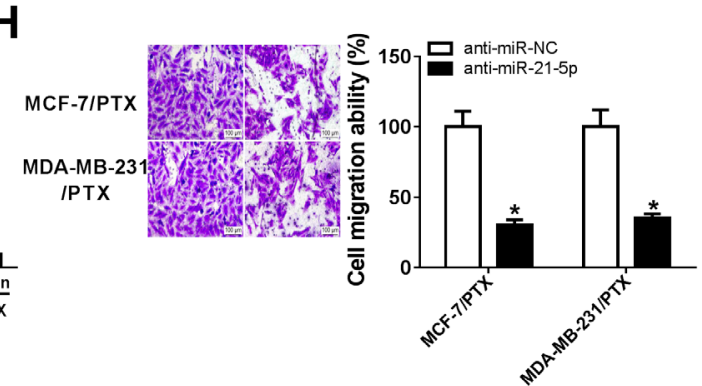

J
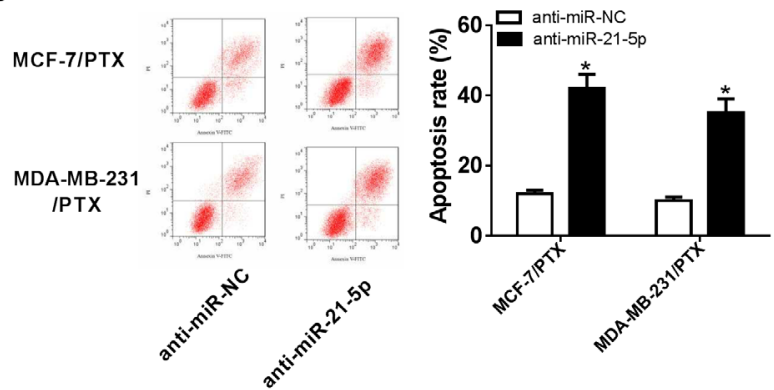

Figure 2. MiR-21-5p silencing ameliorated PTX resistance and inhibited the progression in PTX-resistant BC cell lines. A) MCF-7/PTX and MDAMB-231/PTX cells were transfected with anti-miR-NC or anti-miR-21-5p, followed by the detection of miR-21-5p expression. B) Transfected cells

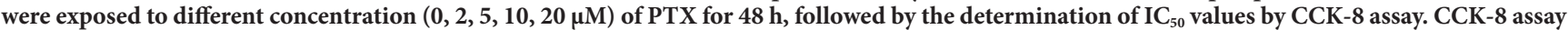
of cell proliferation ability in transfected MCF-7/PTX (C) and MDA-MB-231/PTX (D) cells. E) Flow cytometry analysis of cell cycle progression in transfected cells. (F and G) Western blotting analysis of vimentin and E-cadherin levels in transfected cells. Transwell assays of cell migration ( $H)$ and invasion (I) in transfected cells. (J) Flow cytometry analysis of cell apoptosis in transfected cells. ${ }^{*} \mathrm{p}<0.05$ vs. anti-miR-NC. 
miR-21-5p and PDCD4 expression levels in MCF-7/PTX, MDA-MB-231/PTX cells and their parental cells. qRT-PCR data presented that miR-21-5p was strikingly upregulated in MCF-7 and MDA-MB-231 cells compared to MCF-10A cells (Figure 1E). Moreover, PTX induction resulted in a more distinct promotion on miR-21-5p expression in MCF-7/ PTX and MDA-MB-231/PTX cells (Figure 1E). Conversely, PDCD4 expression was markedly downregulated in PTX-resistant BC cell lines (Figure 1F).

MiR-21-5p silencing ameliorated PTX resistance and inhibited the progression in PTX-resistant BC cell lines. Then, loss-of-function experiments were performed by transfecting anti-miR-21-5p into MCF-7/PTX and MDA-MB231/PTX cells. As shown in Figure 2A, the expression of miR-21-5p was highly decreased by transfection with antimiR-21-5p in MCF-7/PTX and MDA-MB-231/PTX cells. Subsequently, transfected cells were exposed to different concentration of PTX. CCK- 8 assay revealed that miR-21-5p silencing in PTX-resistant cell lines resulted in a lower $\mathrm{IC}_{50}$ values of PTX (Figure 2B). Further, miR-21-5p silencing repressed cell proliferation (Figures $2 \mathrm{C}$ and $2 \mathrm{D}$ ) and cell cycle progression (Figure 2E) compared with corresponding control. Western blotting analysis revealed that miR-21-5p silencing led to a decrease of vimentin level and an increase of E-cadherin expression, indicating that miR-21-5p silencing repressed the epithelial-mesenchymal transition (EMT) of MCF-7/PTX and MDA-MB-231/PTX cells (Figures 2F and $2 \mathrm{G}$ ). Additionally, miR-21-5p silencing highly inhibited the migration (Figure 2H) and invasion (Figure 2I) and promoted the apoptosis of MCF-7/PTX and MDA-MB-231/ PTX cells (Figure 2J).

PDCD4 was a direct target of miR-21-5p in PTX-resistant BC cell lines. Previous reports verified that PDCD4 was a functional target of miR-21 in BC and colorectal cancer cells $[15,16]$. Here, we determined whether PDCD4 was a direct target of miR-21-5p in PTX-resistant BC cell lines. Thus, dualluciferase reporter assays were performed by co-transfection with PDCD4 3'-UTR-WT or PDCD4 3'-UTR-MUT into MCF-7/PTX and MDA-MB-231/PTX cells, together with miR-NC mimics, miR-21-5p mimics, anti-miR-NC orantimiR-21-5p. Results showed that the luciferase activities of PDCD4 3'-UTR-WT were significantly weakened by introduction with miR-21-5p mimics, while they were markedly enhanced in the presence of anti-miR-21-5p (Figures 3A-D). Whereas no change was found in the luciferase activities of PDCD4 3'-UTR-MUT in response to the alteration of
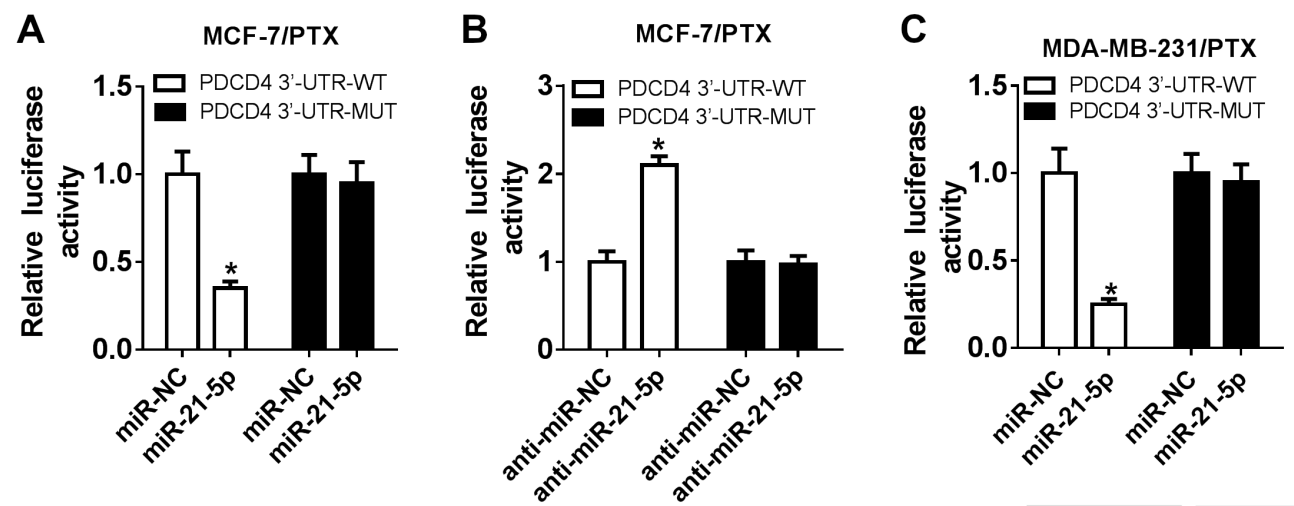

D

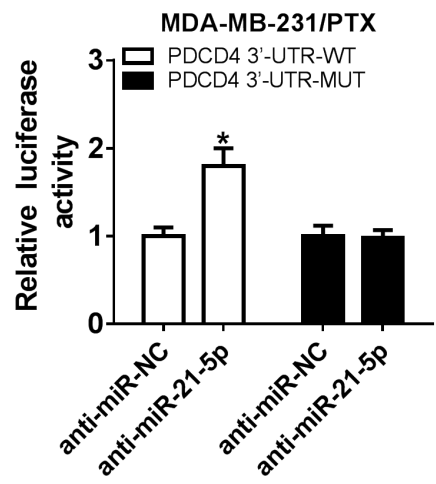

E

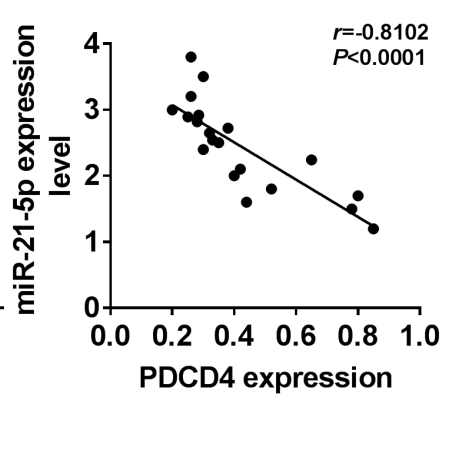

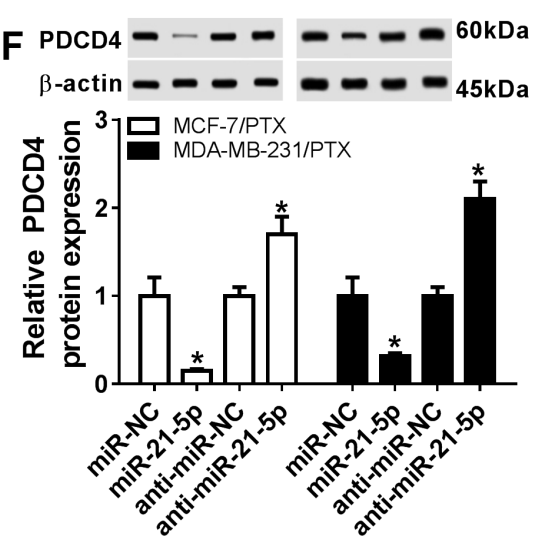

Figure 3. PDCD4 was a direct target of miR-21-5p in PTX-resistant BC cell lines. A-D) Luciferase reporter assay was performed by co-transfection with PDCD4 3'-UTR-WT or PDCD4 3'-UTR-MUT into MCF-7/PTX and MDA-MB-231/PTX cells together with miR-NC mimics, miR-21-5p mimics, anti-miR-NC and anti-miR-21-5p. E) The correlation between PDCD4 expression and miR-21-5p level was determined in BC tissues. F) Western blotting analysis of PDCD4 expression in MCF-7/PTX and MDA-MB-231/PTX cells transfected with miR-NC mimics, miR-21-5p mimics, anti-miR-NC or anti-miR-21-5p. ${ }^{*} \mathrm{p}<0.05$ vs. miR-NC or anti-miR-NC. 
miR-21-5p expression (Figures 3A-D). More interestingly, we found that PDCD4 level was inversely correlated with miR-21-5p expression in BC tissues (Figure 3E). Further, we observed whether miR-21-5p regulated PDCD4 expression in PTX-resistant BC cells. These data revealed that compared with respective control, PDCD4 expression was significantly inhibited by transfection of miR-21-5p mimics, while it was highly promoted following miR-21-5p silencing in MCF-7/ PTX and MDA-MB-231/PTX cells (Figure 3F).

PDCD4 overexpression reduced PTX resistance and repressed the progression in PTX-resistant $\mathrm{BC}$ cell lines. Further, we explored the effect of PDCD4 on PTX resistance and progression of PTX-resistant cell lines by transfecting with pcDNA-NC or pcDNA-PDCD4. These results revealed that pcDNA-PDCD4 transfection resulted in a marked increase of PDCD4 mRNA and protein levels in MCF-7/PTX and MDA-MB-231/PTX cells (Figures 4A and 4B). Then, transfected cells were treated with different concentration of PTX. CCK-8 assay demonstrated that PDCD4 overex- pression highly decreased the $\mathrm{IC}_{50}$ values of PTX (Figure 4C). Subsequently, functional experiments indicated that PDCD4 upregulation resulted in a suppression of cell proliferation (Figures 4D and 4E), cell cycle progression (Figure 4F), EMT (Figures $4 \mathrm{G}$ and $4 \mathrm{H}$ ), migration and invasion capacities (Figures $4 \mathrm{I}$ and $4 \mathrm{~J}$ ), as well as an enhancement of cell apoptosis in MCF-7/PTX and MDA-MB-231/PTX cells (Figure $4 \mathrm{~K}$ ).

MiR-21-5p exerted its regulatory effect by PDCD4 in PTX-resistant BC cell lines. To further investigate whether miR-21-5p exerting its regulatory effect on PTX resistance and progression in PTX-resistant cell lines was mediated by PDCD4, anti-miR-21-5p was transfected into PTX-resistant cell lines together with si-PDCD4. Western blotting analysis revealed that compared to negative control, transfection of si-PDCD4 highly antagonized the promotion effect of anti-miR-21-5p on PDCD4 expression in MCF-7/PTX and MDA-MB-231/PTX cells (Figure 5A). Moreover, CCK-8 assay demonstrated that anti-miR-21-5p-mediated decreased
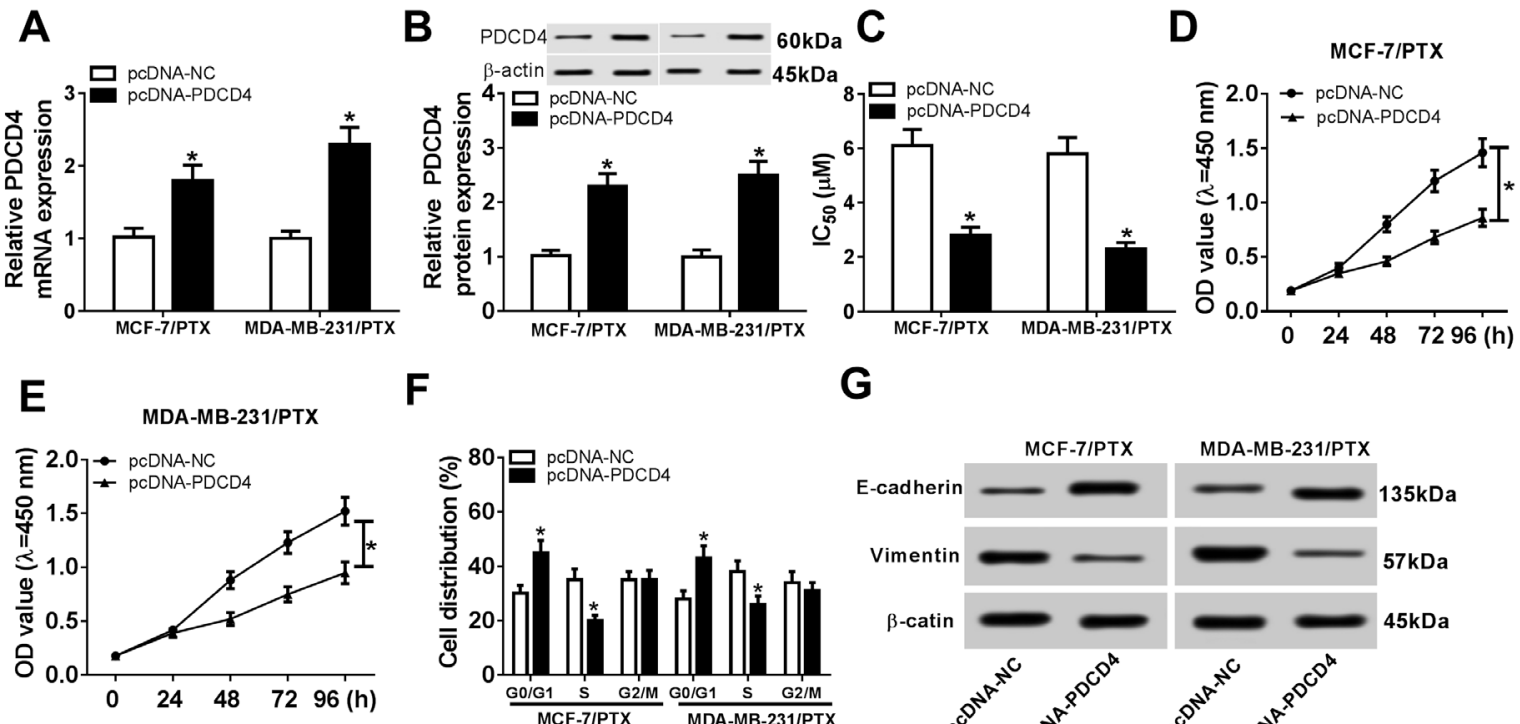

$\mathbf{F}$

G
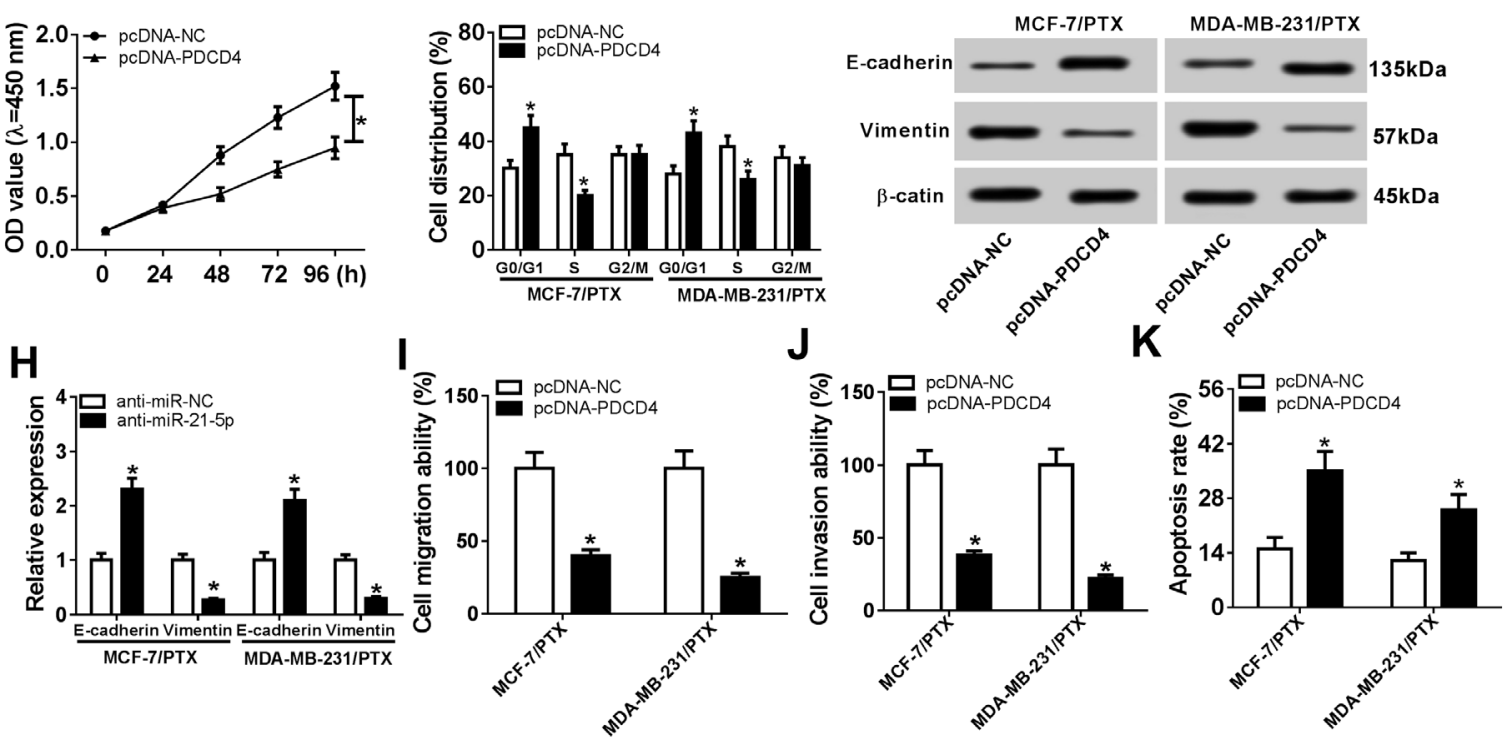

Figure 4. The effect of PDCD4 on PTX resistance and progression of PTX-resistant BC cell lines. MCF-7/PTX and MDA-MB-231/PTX cells were transfected with pcDNA-NC or pcDNA-PDCD4. qRT-PCR assay of PDCD4 mRNA level (A) and western blotting analysis of PDCD4 protein expression (B) in transfected cells. C) Transfected cells were exposed to different concentration $(0,2,5,10,20 \mu \mathrm{M})$ of PTX for $48 \mathrm{~h}$, followed by the measurement of $\mathrm{IC}_{50}$ values of PTX by CCK-8 assay. D and E) CCK-8 assay of the proliferation ability in transfected cells. F) Flow cytometry analysis of cell cycle progression in treated cells. G and $\mathrm{H}$ ) Western blotting analysis of E-cadherin and vimentin levels in treated cells. Transwell assays of cell migration (I) and invasion (J) capacities in transfected cells. K) Flow cytometry analysis of cell apoptosis in transfected cells. ${ }^{*} \mathrm{p}<0.05 \mathrm{vs}$. pcDNA-NC. 


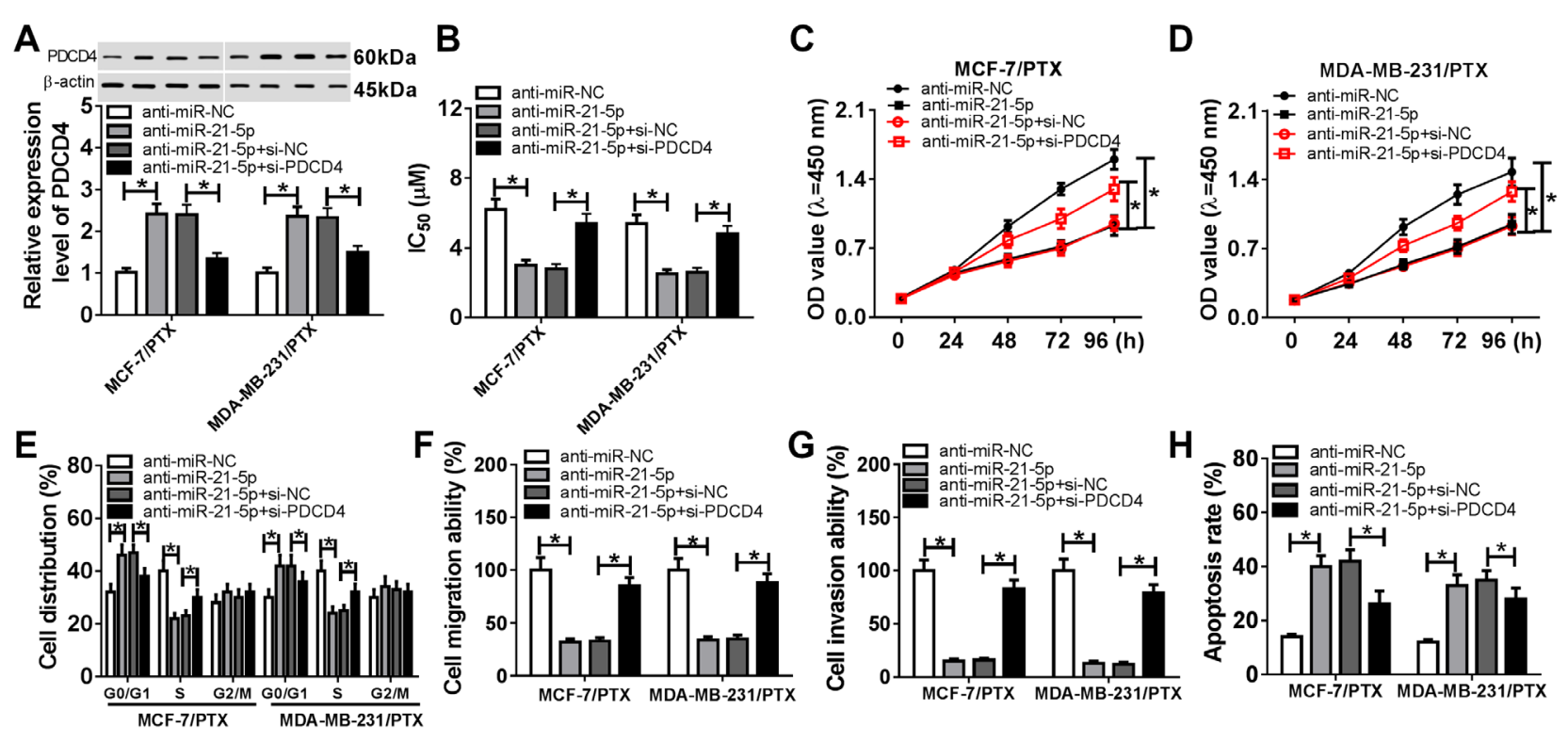

Figure 5. MiR-21-5p exerted its regulatory effect by PDCD4. MCF-7/PTX and MDA-MB-231/PTX cells were transfected with anti-miR-NC, antimiR-21-5p, anti-miR-21-5p+si-NC or anti-miR-21-5p+si-PDCD4, followed by the detection of PDCD4 expression by western blotting analysis (A), $\mathrm{IC}_{50}$ values of PTX by CCK-8 assay (B), cell proliferation ability by CCK-8 assay (C) and (D), cell cycle progression by flow cytometry analysis (E), cell migration and invasion capacities by Transwell assay (F) and (G), and cell apoptosis by flow cytometry analysis (H). ${ }^{*} \mathrm{p}<0.05 \mathrm{vs}$. anti-miR-NC or antimiR-21-5p+si-NC.

A

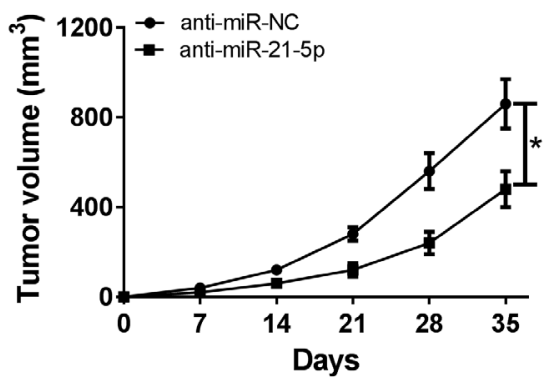

C

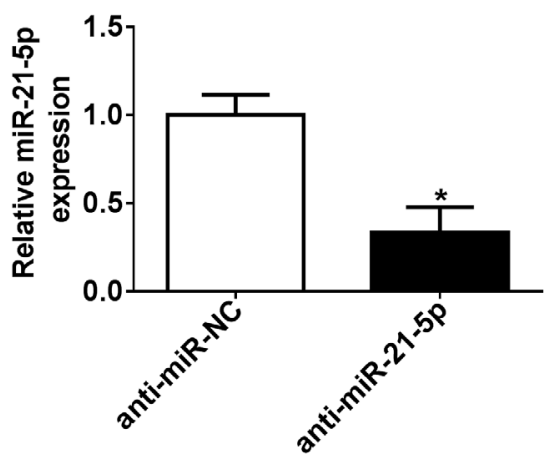

B

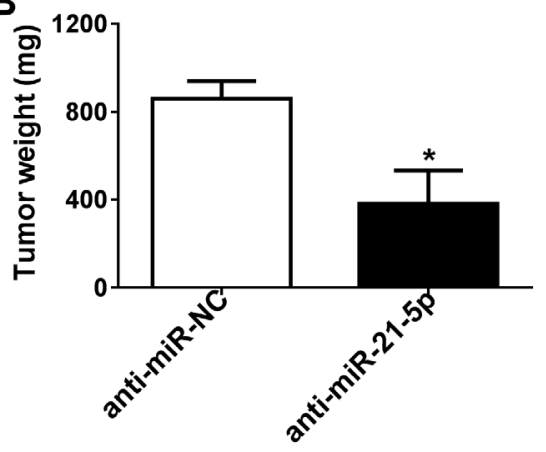

D
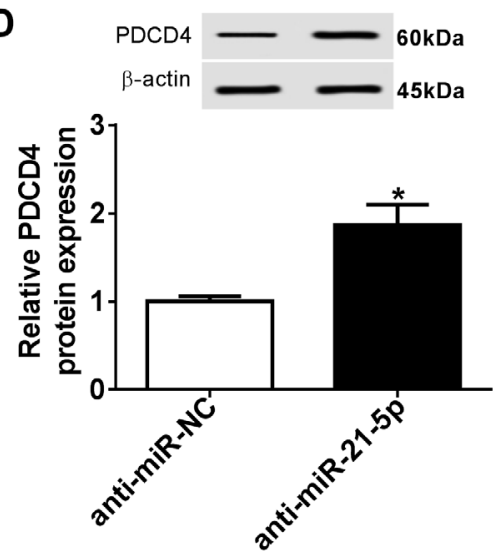

Figure 6. MiR-21-5p silencing inhibited tumor growth in vivo. MCF-7/PTX cells $\left(5.0 \times 10^{6}\right)$ were subcutaneously injected into nude mice. 2 days after injection, intratumoral injection of anti-miR-NC or anti-miR-21-5p was performed every 4 days $(n=10)$. Also, 5 days after injection, an intravenous administration of PTX solution $(1 \mathrm{mg} / \mathrm{kg})$ in each mouse was performed every 5 days. All mice were euthanized 35 days after injection. A) Tumor volume was measured every one week by a calipers. B) Tumor average weight was calculated. C) qRT-PCR assay of miR-21-5p expression in excised tumor tissues. D) Western blotting analysis of PDCD4 level in xenograft tissues. ${ }^{*} \mathbf{p}<0.05$ vs. anti-miR-NC. 
IC $_{50}$ of PTX was strikingly abolished by co-transfection with si-PDCD4 (Figure 5B). In parallel, functional experiments indicated that PDCD4 expression restoration remarkably antagonized the regulatory effect of miR-21-5p silencing on cell proliferation, cell cycle progression, migration, invasion and apoptosis (Figures $5 \mathrm{C}-\mathrm{H}$ ).

MiR-21-5p silencing inhibited tumor growth in vivo. In order to further validate the important role of miR-21-5p as a regulator on PTX-resistance BC cell lines, we constructed mice xenograft models of $\mathrm{BC}$ by subcutaneous injection with MCF-7/PTX cells. Results demonstrated that compared with negative control, miR-21-5p silencing significantly inhibited tumor growth, indicated by the decrease of tumor volume (Figure 6A) and tumor weight (Figure 6B). Moreover, miR-21-5p silencing resulted in a decrease of miR-21-5p expression and an increase of PDCD4 level in xenograft tissues (Figures 6C and 6D).

\section{Discussion}

MiRNAs have been elucidated as oncogenes or tumor suppressors involved in tumor growth, metastasis, apoptosis and chemoresistance [5]. A large number of miRNAs play pivotal roles in human cancers, including BC. For instance, miR-10b overexpression promoted the invasion and metastasis in non-metastatic BC cells [19]. Upregulation of miR-155 contributed to tumor angiogenesis through targeting VHL and associated with poor prognosis in triple-negative BC [20]. Conversely, miR-125b overexpression suppressed the EMT by Sema4C in PTX-resistant BC cells [21]. In addition, upregulation of miR-24 and miR-125b sensitized PTX-resistant BC cells to PTX [21, 22]. MiR-451 also influenced the drug resistance of PTX-resistant BC cells by enhancing cell apoptosis [23].

In our study, we validated that miR-21-5p was upregulated in BC tissues and PTX-resistant BC cell lines, highlighting its role as important regulator in PTX-resistant BC. MiR-21-5p was overexpressed and displayed oncogenic activity in most cancers, such as clear cell renal cell carcinoma [24], rectal cancer [25], laryngeal squamous cell carcinoma [26] and BC [15]. Also, miR-21 overexpression was identified as predictor of worse prognosis in various carcinomas [27]. Upregulation of miR-21 induced radiation-resistance of non-small cell lung cancer [28]. Moreover, miR-21 contributed to chemoresistance in several cancers $[12,17]$. Further, we verified that miR-21-5p silencing ameliorated PTX resistance and inhibited the progression in PTX-resistant BC cell lines in vitro, and repressed PTX-resistant tumor growth in vivo.

PDCD4, a novel tumor suppressor, is manifested to suppress tissue polypeptide antigen (TPA)-induced neoplastic transformation [29] and translation [30], repress tumorigenesis and progression [13]. Moreover, PDCD4 was identified to suppress IRES-mediated translation of antiapoptotic proteins (such as XIAP and $\mathrm{Bcl}-\mathrm{xl}$ ), suggesting its role as a tumor suppressor [31]. PDCD4 knockdown

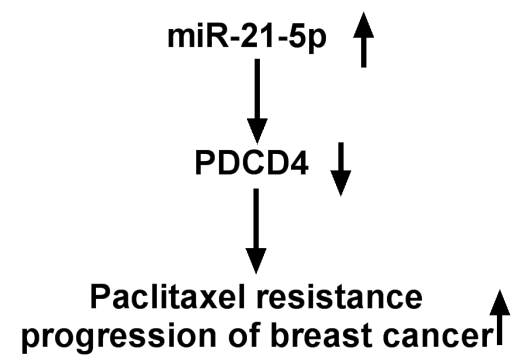

Figure 7. A schematic model of regulation of BC progression and PTXresistance by $\mathrm{miR}-21-5 \mathrm{p}$ regulatory axis.

resulted in a decrease of E-cadherin expression and activated $\beta$-catenin/Tcf-dependent transcription, providing molecular explanation of how PDCD4 represses colon tumor invasion [32]. Additionally, PDCD4 was demonstrated to act as a novel and independent prognostic target in colorectal carcinomas [33]. PDCD4 was also verified to be associated with drug resistance in cancers [14]. In the present study, our data indicated that PDCD4 was downregulated in BC tissues and PTX-resistant BC cell lines. Moreover, upregulation of PDCD4 reduced PTX resistance and repressed the progression in PTX-resistant BC cell lines.

Increasing evidences showed that PDCD4 was an important functional target of miR-21 in multiple human cancers, such as gastric cancer [34], glioblastoma [35] and medullary thyroid carcinoma [36]. The axis of miR-21 targeting PDCD4 was illuminated to be relevant to chemoresistance in pancreatic cancer [17], androgen-independent prostate cancer [37] and tongue squamous cell carcinoma [38]. Furthermore, miR-21 depletion inhibited the growth of MCF-7 BC cells through targeting PDCD4, indicating miR-21/PDCD4 axis in BC [15]. Therefore, we validated whether miR-21-5p exerting its regulatory function on PTX-resistant BC cell lines was mediated by PDCD4. As expected, PDCD4 was confirmed to be a direct target of miR-21-5p in PTX-resistant BC cell lines. Moreover, miR-21-5p exerted its regulatory effect on PTX resistance, progression and tumor growth by PDCD4 in PTX-resistant BC cell lines.

In conclusion, our study indicated that miR-21-5p silencing ameliorated PTX resistance and inhibited the progression in PTX-resistant BC cell lines at least partly by targeting PDCD4, providing miR-21-5p as an effective therapeutic target for PTX-resistant BC treatment.

\section{References}

[1] DESANTIS C, MA J, BRYAN L, JEMAL A. Breast cancer statistics, 2013. CA Cancer J Clin 2014; 64: 52-62. https:// doi.org/10.3322/caac. 21203

[2] BERRY DA, CRONIN KA, PLEVRITIS SK, FRYBACK DG, CLARKE $L$ et al. Effect of screening and adjuvant therapy on mortality from breast cancer. N Engl J Med 2005; 353: 1784-1792. https://doi.org/10.1056/NEJMoa050518 
[3] GONZALEZ-ANGULO AM, MORALES-VASQUEZ F HORTOBAGYI GN. Overview of resistance to systemic therapy in patients with breast cancer. Adv Exp Med Biol 2007; 608:1-22. https://doi.org/10.1007/978-0-387-74039-3_1

[4] HAMMOND SM. An overview of microRNAs. Adv Drug Deliv Rev 2015; 87: 3-14. https://doi.org/10.1016/j. addr.2015.05.001

[5] HAYES J, PERUZZI PP, LAWLER S. MicroRNAs in cancer: biomarkers, functions and therapy. Trends Mol Med 2014; 20: 460-469. https://doi.org/10.1016/j.molmed.2014.06.005

[6] XIA Y, WANG Y, WANG Q, GHAFFAR M, WANG Y et al. Increased miR-203-3p and reduced miR-21-5p synergistically inhibit proliferation, migration, and invasion in esophageal cancer cells. Anticancer Drugs 2018; 30: 38-45. https:// doi.org/10.1097/cad.0000000000000687

[7] YUAN Y, NIU F, NOLTE IM, KOERTS J, DE JONG D et al. MicroRNA High Throughput Loss-of-Function Screening Reveals an Oncogenic Role for miR-21-5p in Hodgkin Lymphoma. Cell Physiol Biochem 2018; 49: 144-159. https://doi. org/10.1159/000492850

[8] SEIKE M, GOTO A, OKANO T, BOWMAN ED, SCHETTER AJ et al. MiR-21 is an EGFR-regulated anti-apoptotic factor in lung cancer in never-smokers. Proc Natl Acad Sci U S A 2009; 106: 12085-12090. https://doi.org/10.1073/ pnas.0905234106

[9] YU W, ZHU K, WANG Y, YU H, GUO J. Overexpression of miR-21-5p promotes proliferation and invasion of colon adenocarcinoma cells through targeting CHL1. Mol Med 2018; 24:36. https://doi.org/10.1186/s10020-018-0034-5

[10] QIAN B, KATSAROS D, LU L, PRETI M, DURANDO A et al. High miR-21 expression in breast cancer associated with poor disease-free survival in early stage disease and high TGF-beta1. Breast Cancer Res Treat 2009; 117: 131-140. https://doi.org/10.1007/s10549-008-0219-7

[11] ZHAO Y, ZHAO L, ISCHENKO I, BAO Q, SCHWARZ B et al. Antisense inhibition of microRNA-21 and microRNA-221 in tumor-initiating stem-like cells modulates tumorigenesis, metastasis, and chemotherapy resistance in pancreatic cancer. Target Oncol 2015; 10: 535-548. https://doi.org/10.1007/ s11523-015-0360-2

[12] MENG F, HENSON R, LANG M, WEHBE H, MAHESHWARI $S$ et al. Involvement of human micro-RNA in growth and response to chemotherapy in human cholangiocarcinoma cell lines. Gastroenterology 2006; 130: 2113-2129. https://doi.org/10.1053/j.gastro.2006.02.057

[13] MA G, ZHANG H, DONG M, ZHENG X, OZAKI I et al. Downregulation of programmed cell death 4 (PDCD4) in tumorigenesis and progression of human digestive tract cancers. Tumour Biol 2013; 34: 3879-3885. https://doi. org/10.1007/s13277-013-0975-9

[14] XU H, DEPHOURE N, SUN H, ZHANG H, FAN F et al. Proteomic Profiling of Paclitaxel Treated Cells Identifies a Novel Mechanism of Drug Resistance Mediated by PDCD4. J Proteome Res 2015; 14: 2480-2491. https://doi.org/10.1021/acs. jproteome.5b00004

[15] FRANKEL LB, CHRISTOFFERSEN NR, JACOBSEN A, LINDOW M, KROGH A et al. Programmed cell death 4 (PDCD4) is an important functional target of the microRNA miR-21 in breast cancer cells. J Biol Chem 2008; 283: 10261033. https://doi.org/10.1074/jbc.M707224200
[16] ASANGANI IA, RASHEED SA, NIKOLOVA DA, LEUPOLD JH, COLBURN NH et al. MicroRNA-21 (miR-21) post-transcriptionally downregulates tumor suppressor $\operatorname{Pdcd} 4$ and stimulates invasion, intravasation and metastasis in colorectal cancer. Oncogene 2008; 27: 2128-2136. https:// doi.org/10.1038/sj.onc.1210856

[17] WEI X, WANG W, WANG L, ZHANG Y, ZHANG X et al. MicroRNA-21 induces 5-fluorouracil resistance in human pancreatic cancer cells by regulating PTEN and PDCD4. Cancer Med 2016; 5: 693-702. https://doi.org/10.1002/ cam 4.626

[18] SPROUSE AA, HERBERT BS. Resveratrol augments paclitaxel treatment in MDA-MB-231 and paclitaxel-resistant MDA-MB-231 breast cancer cells. Anticancer Res 2014; 34: 5363-5374.

[19] MA L, TERUYA-FELDSTEIN J, WEINBERG RA. Tumour invasion and metastasis initiated by microRNA-10b in breast cancer. Nature 2007; 449: 682-688. https://doi.org/10.1038/ nature 06174

[20] KONG W, HE L, RICHARDS EJ, CHALLA S, XU CX et al. Upregulation of miRNA-155 promotes tumour angiogenesis by targeting VHL and is associated with poor prognosis and triple-negative breast cancer. Oncogene 2014; 33: 679-689. https://doi.org/10.1038/onc.2012.636

[21] YANG Q, WANG Y, LU X, ZHAO Z, ZHU L et al. MiR-125b regulates epithelial-mesenchymal transition via targeting Sema4C in paclitaxel-resistant breast cancer cells. Oncotarget 2015; 6: 3268-3279. https://doi.org/10.18632/oncotarget. 3065

[22] GONG JP, YANG L, TANG JW, SUN P, HU Q et al. Overexpression of microRNA-24 increases the sensitivity to paclitaxel in drug-resistant breast carcinoma cell lines via targeting ABCB9. Oncol Lett 2016; 12: 3905-3911. https://doi. org/10.3892/ol.2016.5139

[23] GU X, LI JY, GUO J, LI PS, ZHANG WH. Influence of MiR451 on Drug Resistances of Paclitaxel-Resistant Breast Cancer Cell Line. Med Sci Monit 2015; 21: 3291-3297. https:// doi.org/10.12659/MSM.894475

[24] KOWALCZYK AE, KRAZINSKI BE, GODLEWSKI J, GRZEGRZOLKA J, KIEWISZ J et al. SATB1 is Down-regulated in Clear Cell Renal Cell Carcinoma and Correlates with miR-21-5p Overexpression and Poor Prognosis. Cancer Genomics Proteomics 2016; 13: 209-217.

[25] LOPES-RAMOS CM, HABR-GAMA A, QUEVEDO BDE $\mathrm{S}$, FELICIO NM, BETTONI F et al. Overexpression of miR21-5p as a predictive marker for complete tumor regression to neoadjuvant chemoradiotherapy in rectal cancer patients. BMC Med Genomics 2014; 7: 68. https://doi.org/10.1186/ s12920-014-0068-7

[26] RE M, MAGLIULO G, GIOACCHINI FM, BAJRAKTARI A, BERTINI A et al. Expression Levels and Clinical Significance of miR-21-5p, miR-let-7a, and miR-34c-5p in Laryngeal Squamous Cell Carcinoma. Biomed Res Int 2017; 2017: 3921258. https://doi.org/10.1155/2017/3921258

[27] ZHU W, XU B. MicroRNA-21 identified as predictor of cancer outcome: a meta-analysis. PLoS One 2014; 9: e103373. https://doi.org/10.1371/journal.pone.0103373 
[28] WANG XC, WANG W, ZHANG ZB, ZHAO J, TAN XG et al. Overexpression of miRNA-21 promotes radiation-resistance of non-small cell lung cancer. Radiat Oncol 2013; 8: 146. https://doi.org/10.1186/1748-717x-8-146

[29] YANG HS, JANSEN AP, NAIR R, SHIBAHARA K, VERMA AK et al. A novel transformation suppressor, Pdcd4, inhibits AP-1 transactivation but not NF-kappaB or ODC transactivation. Oncogene 2001; 20: 669-676. https://doi. org/10.1038/sj.onc. 1204137

[30] WEDEKEN L, SINGH P, KLEMPNAUER KH. Tumor suppressor protein Pdcd4 inhibits translation of p53 mRNA. J Biol Chem 2011; 286: 42855-42862. https://doi.org/10.1074/ jbc.M111.269456

[31] LIWAK U, THAKOR N, JORDAN LE, ROY R, LEWIS SM et al. Tumor suppressor PDCD4 represses internal ribosome entry site-mediated translation of antiapoptotic proteins and is regulated by S6 kinase 2. Mol Cell Biol 2012; 32: 18181829. https://doi.org/10.1128/mcb.06317-11

[32] WANG Q, SUN ZX, ALLGAYER H, YANG HS. Downregulation of E-cadherin is an essential event in activating betacatenin/Tcf-dependent transcription and expression of its target genes in Pdcd4 knockdown cells. Oncogene 2010; 29: 128-138. https://doi.org/10.1038/onc.2009.302

[33] ALLGAYER H. Pdcd4, a colon cancer prognostic that is regulated by a microRNA. Crit Rev Oncol Hematol 2010; 73: 185-191. https://doi.org/10.1016/j.critrevonc.2009.09.001
[34] MOTOYAMA K, INOUE H, MIMORI K, TANAKA F, KOJIMA $\mathrm{K}$ et al. Clinicopathological and prognostic significance of PDCD4 and microRNA-21 in human gastric cancer. Int J Oncol 2010; 36: 1089-1095. https://doi.org/10.3892/ ijo_00000590

[35] GAUR AB, HOLBECK SL, COLBURN NH, ISRAEL MA. Downregulation of Pdcd 4 by mir-21 facilitates glioblastoma proliferation in vivo. Neuro Oncol 2011; 13: 580-590. https:// doi.org/10.1093/neuonc/nor033

[36] PENNELLI G, GALUPPINI F, BAROLLO S, CAVEDON E, BERTAZZA L et al. The PDCD4/miR-21 pathway in medullary thyroid carcinoma. Hum Pathol 2015; 46: 50-57. https:// doi.org/10.1016/j.humpath.2014.09.006

[37] SHI GH, YE DW, YAO XD, ZHANG SL, DAI B et al. Involvement of microRNA-21 in mediating chemo-resistance to docetaxel in androgen-independent prostate cancer PC3 cells. Acta Pharmacol Sin 2010; 31: 867-873. https://doi. org/10.1038/aps.2010.48

[38] REN W, WANG X, GAO L, LI S, YAN X et al. MiR-21 modulates chemosensitivity of tongue squamous cell carcinoma cells to cisplatin by targeting PDCD4. Mol Cell Biochem 2014; 390: 253-262. https://doi.org/10.1007/s11010-0141976-8 